\title{
Biologia de Centris Fabricius (Hymenoptera, Anthophoridae, Centridini) em matas contínuas e fragmentos na Amazônia Central
}

\author{
Elder F. Morato ${ }^{1}$ \\ Marcos V. B. Garcia ${ }^{2}$ \\ Lúcio Antonio de O. Campos ${ }^{3}$
}

\begin{abstract}
Biology of Centris Fabricius (Hymenoptera, Anthophoridae, Centridini) in continuous forest and fragments in Central Amazonia. A study of four tree-hole nesting bee species of Centris Fabricius, 1804 was conducted between July, 1988 and June, 1990 in isolated and continuous "terra firme" forests at Manaus region. Nests were obtained from wood trap-nests of different diameters and consisted of a linear series of brood cells. Centris dichrootricha (Moure, 1945) and C. terminata Smith, 1874 were the most abundant species. Centris terminata nested principally in gaps and continuous forest and $C$. dichrootricha in cleared areas and small fragments of forest. More nests were obtained in trap-nests placed at 8 and $15 \mathrm{~m}$ height above ground. Nesting activity was more pronounced in the wet season between August and January. Natural enemies are the beetle Tetraonyx Latreille, 1805 and the bees Coelioxys Latreille, 1809, Mesocheira Lepeletier \& Serville, 1825 and Mesoplia Lepeletier, 1841. Nest architecture of Centris spp. are described.

KEY WORDS. Anthophoridae, Centris, Amazonia, forest fragmentation, bee biology
\end{abstract}

Abelhas do gênero Centris Fabricius, 1804 (Anthophoridae) variam muito quanto aos sítios usados para nidificação (MICHENER \& LANGE 1958; CovILLE et al. 1983). Espécies dos subgenêros Trachina Klug, 1807, Centris Fabricius, 1804, Paracentris Cameron, 1903, Xerocentris Snelling, 1974 e Melanocentris Friese, 1900 escavam seus ninhos no chão ou em barrancos (VEZEY-FITZGERALD 1939; AlCOCK et al. 1976; BATRA \& SCHUSTER 1977; VINSON \& FRANKIE 1977, 1988, 1991; RAW 1984; COVILlE et al. 1983, 1986; GOTTSBERGER et al. 1988) e espécies do subgenêro Ptilotopus Klug, 1810 escavam seus ninhos em termiteiros (PICKEL 1928; VEZEY-FITZGERALD 1939; BENNETT 1964). Por outro lado, as espécies dos subgenêros Hemisiella Moure, 1945 e Heterocentris Cockerell, 1899 constroem seus ninhos em cavidades preexistentes tais como galerias em madeira feitas por outros insetos ou ninhos abandonados de outras espécies de abelhas e vespas (RAU

1) Departamento de Ciências da Natureza, Universidade Federal do Acre. 69915-900 Rio Branco, Acre, Brasil.

E-mail: morato@mono.icb.ufmg.br

2) EMBRAPA-CPAA. Caixa Postal 319, 69048-660 Manaus, Amazonas, Brasil.

E-mail: mgarcia@internext.com.br

3) Departamento de BiologiaGeral, Universidade Federal de Viçosa. 36571-000 Viçosa, Minas Gerais, Brasil.

E-mail: Icampos@email.ufv.br 
1943; MiCHENER \& LANGE 1958; CHANDLER et al. 1985); utilizam também sítios artificiais tais como peças de madeira perfuradas oferecidas como "ninhos-armadilhas", o que facilita o estudo de seus hábitos de nidificação.

FRANKIE et al. $(1988,1993)$ usando ninhos-armadilhas estudaram, quanto a preferência por diferentes habitats, C. (Hemisiella) nitida Smith, 1874, C. (Hemisiella) trigonoides Lepeletier, 1841, C. (Hemisiella) vittata Lepeletier, 1841, C. (Heterocentris) analis (Fabricius, 1804) e C. (Heterocentris) bicornuta Mocsary, 1899 em áreas de matas na Costa Rica.

Os efeitos do isolamento de porções de mata tropical vêm sendo estudados na Amazônia Central, em região próxima de Manaus, pelo Projeto Dinâmica Biológica de Fragmentos Florestais (PDBFF). Conforme resultados do PDBFF esse isolamento tem produzido grandes modificações na fauna, flora e microclima da floresta (LOVEJOY et al. 1986).

Este trabalho teve como objetivo, dentro do contexto do PDBFF, estudar aspectos da biologia de espécies de Centris que nidificam em ninhos-armadilhas em matas contínuas, fragmentos e áreas desmatadas, em uma região que tem sofrido intenso desmatamento próxima a Manaus, Amazonas.

\section{MATERIAL E MÉTODOS}

As coletas foram realizadas no período de julho de 1988 a junho de $1990 \mathrm{em}$ áreas de florestas de terra firme (PIRES \& PRANCE 1985) localizadas a $90 \mathrm{~km}$ ao norte de Manaus, a $59^{\circ} 52^{\prime} \mathrm{W}$ e $2^{\circ} 24^{\prime} \mathrm{S}$, em áreas de estudo do PDBFF. A região apresenta vegetação de floresta tropical úmida de terra firme, com dossel atingindo em média 30-37 m de altura (BIERREGAARD \& LOVEJOY 1988). O sub-bosque é aberto e possui um grande número de palmeiras acaules. Os solos são, em geral, do tipo latossolos e pobres em nutrientes. O relevo é plano-ondulado, recortado por inúmeros igarapés. A região se caracteriza por apresentar uma estação chuvosa entre dezembro e maio e uma estação seca entre junho e novembro (RIBEIRO \& ADIS 1984). Dados mensais de precipitação e temperatura da região durante o período de coleta foram obtidos na Estação Meteorológica da Embrapa, situada no km 28 da Rodovia AM-010 (59 $59^{\prime} \mathrm{W}$ e 2 $2^{\circ} 52^{\prime} \mathrm{S}$ ).

Ninhos-armadilhas foram feitos com peças de madeira de $25 \times 35 \times 120 \mathrm{~mm}$, perfuradas de modo a formar orifícios de 4,8; 9,5 e 12,7 mm de diâmetro e $80 \mathrm{~mm}$ de profundidade. A madeira utilizada foi Pithecellobium Martius (Leguminosae) por ser inodora e não possuir fibras que obstruiriam a cavidade.

As coletas foram realizadas em dois fragmentos de mata de 1 ha, dois fragmentos de 10 ha, um fragmento de 100 ha, todos de mata nativa preservada, em sete locais de mata contínua (mata não perturbada), 22 clareiras naturais situadas no interior da mata contínua e dois locais onde a vegetação nativa foi cortada (áreas de derrubada). As clareiras tinham área variando entre $25 \mathrm{e} 40 \mathrm{~m}^{2}$ e se originaram da queda natural de árvores. Durante o período de coleta de dados, uma das áreas de derrubada era constituída de pastagem e de vegetação secundária com várias espécies de plantas ruderais. A outra era constituída por vegetação secundária muito desenvolvida e fechada, dominada por espécies de Cecropia Loefl. (Cecropiaceae). 
No interior de cada fragmento isolado ou local de mata contínua, foram escolhidas quatro árvores separadas entre si por uma distância máxima de $50 \mathrm{~m}$. Cada conjunto de quatro árvores, onde foram instaladas as armadilhas, constituiu uma unidade amostral. Ao todo, foram estudadas 12 unidades amostrais na mata. Em cada árvore foram colocados três blocos de ninhos-armadilhas: um bloco a 1,5 $\mathrm{m}$; um bloco a $8 \mathrm{~m}$ e outro a $15 \mathrm{~m}$ de altura. Cada bloco continha nove ninhos-armadilhas, três de cada diâmetro, distribuídos ao acaso. Os blocos foram atados a um fio de náilon que passava por uma roldana de metal presa à árvore, a $15 \mathrm{~m}$ de altura. Isso possibilitou a descida, verificação e suspensão dos blocos periodicamente.

Aproximadamente a cada 15 dias as armadilhas foram inspecionadas, e aquelas contendo ninhos foram retiradas sendo substituídas por peças vazias. No laboratório estes ninhos foram abertos e descritos. Após a descrição, os ninhos foram colocados em local apropriado até a emergência dos adultos para identificação das espécies. Em ninhos, dos quais não ocorreram emergências, as espécies foram identificadas, por comparação com ninhos semelhantes, previamente identificados.

Foi calculada a dissimilaridade entre as espécies de abelhas em relação às épocas de fundação de ninhos ao longo dos dois anos de coleta. Para isso, foi utilizado o índice de porcentagem de dissimilaridade (PD) (LUDWIG \& REYNOLDS 1988).

\section{RESULTADOS}

Foram fundados 228 ninhos de quatro espécies de Centris: C. (Heterocentris) terminata Smith, 1874 (56,6\%); C. (Hemisiella) dichrootricha (Moure, 1945) (35,1\%); C. (Heterocentris) analis (Fabricius, 1804) (7\%) e C. (Heterocentris) bicornuta Mocsary, 1899 (1,3\%). Devido a sua maior representatividade serão abordados principalmente os resultados com relação às três primeiras espécies.

\section{Atividade de nidificação em diferentes ambientes}

$\mathrm{O}$ número de ninhos fundados em diferentes ambientes por $C$. terminata $\mathrm{e}$ C. dichrootricha é mostrado na figura 1. C. terminata fundou ninhos em todos os tipos de ambiente. Em clareiras naturais e na mata contínua o número de ninhos foi maior que nos fragmentos de mata e nos locais de derrubada. O número de fundações no interior de clareiras naturais foi significativamente maior que na mata contínua $\left(X^{2}=4,2 ; p<0,05\right)$. Entre fragmentos de diferentes tamanhos a variação foi pequena.

Centris dichrootricha também fundou ninhos em todos os ambientes. Contudo, o número de ninhos foi maior nos fragmentos de mata de 1 e 10 ha e nos locais de derrubada.

Centris analis ocorreu apenas na derrubada (nove ninhos), fragmentos de 1 ha (2) e na mata contínua (5). Os três ninhos de C. bicornuta foram fundados em um fragmento de 10 ha.

\section{Estratificação na floresta}

$\mathrm{O}$ número de ninhos fundados em diferentes alturas nos fragmentos de mata e na mata contínua é mostrado na tabela I. As espécies de Centris fundaram mais ninhos a $15 \mathrm{~m}$ de altura $\left(X^{2}=9,44 ;\right.$ G.L. $\left.=2 ; \mathrm{p}<0,05\right)$. Cerca de $77 \%$ dos ninhos 
fundados em mata contínua e fragmentos, o foram a 8 ou $15 \mathrm{~m}$ de altura. Para $C$. terminata não houve diferenças significativas entre as alturas com relação ao número de ninhos fundados na mata contínua $\left(X^{2}=0,8 ;\right.$ G.L. $\left.=2 ; \mathrm{p}>0,05\right)$. Nos fragmentos, contudo, maior número de ninhos foi fundado a $1,5 \mathrm{~m} \mathrm{e} 8 \mathrm{~m}\left(X^{2}=7,3\right.$; G.L. $=2 ; \mathrm{p}<0,05)$. Centris dichrootricha fundou mais ninhos a $15 \mathrm{~m}$ de altura nos fragmentos $\left(X^{2}=12,0 ;\right.$ G.L. $\left.=2 ; \mathrm{p}<0,05\right)$ e na mata contínua $\left(X^{2}=14,7 ;\right.$ G.L. $=2$; $\mathrm{p}<0,05)$. Os ninhos de $C$. analis e C. bicornuta foram fundados a 8 e $15 \mathrm{~m}$ de altura.
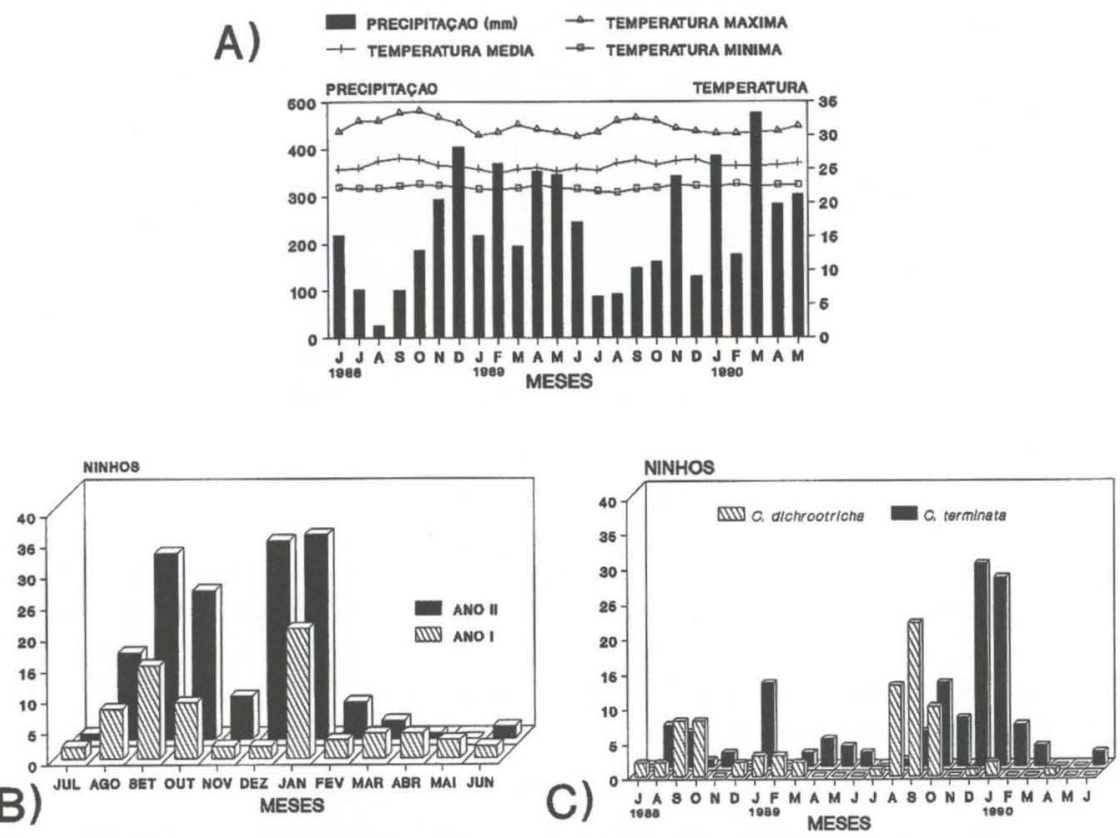

Fig. 1. Número de ninhos coletados na mata contínua (mata cont.), fragmentos isolados de mata $(1,10$ e 100 ha) e outros ambientes entre julho de 1988 a junho de 1990. (A) C. dichrootricha. (B) C. terminata.

Tabela I. Número de ninhos fundados por espécies de Centris em diferentes alturas $(\mathrm{m})$ em fragmentos e na mata contínua, entre junho de 1988 e junho 1990.

\begin{tabular}{|c|c|c|c|c|c|c|c|c|c|}
\hline \multirow[b]{2}{*}{ Espécies $\quad$ Alturas $(\mathrm{m})$} & \multicolumn{3}{|c|}{ Fragmentos } & \multicolumn{3}{|c|}{ Mata contínua } & \multicolumn{3}{|c|}{ Total } \\
\hline & 1,5 & 8,0 & 15,0 & 1,5 & 8,0 & 15,0 & 1,5 & 8,0 & 15,0 \\
\hline Centris terminata & 8 & 14 & 3 & 23 & 19 & 25 & 31 & 33 & 28 \\
\hline Centris dichrootricha & 6 & 12 & 24 & 1 & 4 & 14 & 7 & 16 & 38 \\
\hline Centris analis & & 2 & & & 2 & 3 & & 4 & 3 \\
\hline Centris bicornuta & & 2 & 1 & & & & & 2 & 1 \\
\hline Total & 14 & 30 & 28 & 24 & 25 & 42 & 38 & 55 & 70 \\
\hline
\end{tabular}

$\left({ }^{*}\right)$ Não foram considerados os dados referentes às áreas de derrubada, pois nelas, todos os ninhos-armadilhas foram colocados a $1,5 \mathrm{~m}$ de altura. 


\section{Sazonalidade}

No primeiro ano (julho/88 a junho/89) foram fundados 75 ninhos de Centris e no segundo ano (julho/89 a junho/90) 153. Essa diferença foi significativa $\left(X^{2}=\right.$ $26,7 ; \mathrm{p}<0,001)$. O número de ninhos fundados ao longo dos meses nos dois anos é mostrado na figura $2 \mathrm{~B}$. A atividade de fundação de ninhos foi maior entre os meses de agosto e janeiro ( $84 \%$ dos ninhos fundados). Em ambos os anos, observou-se um pico em setembro e outro em janeiro. Não houve correlação significativa entre os períodos de fundação nos dois anos $\left(\mathrm{r}_{\mathrm{s}}=0,47 ; \mathrm{G} . \mathrm{L} .=10 ; \mathrm{p}>0,05\right)$.

O número de ninhos fundados por $C$. dichrootricha e $C$. terminata ao longo dos meses nos dois anos é mostrado na figura $2 \mathrm{C}$. Para $C$. dichrootricha houve uma correlação significativa entre a atividade de fundação do primeiro para o segundo ano $\left(\mathrm{r}_{\mathrm{s}}=0,62 ; \mathrm{G} . \mathrm{L} .=10 ; \mathrm{p}<0,05\right)$, o que não ocorreu com C. terminata $\left(\mathrm{r}_{\mathrm{s}}=-0,16\right.$; G.L. $=10 ; p>0,05)$. Centris dichrootricha apresentou um pico nos meses de setembro e outubro no primeiro ano e outro em setembro no segundo. Centris terminata apresentou no primeiro ano um pico no mês de janeiro e no segundo um pico mais acentuado nos meses de dezembro e janeiro. Houve uma dissimilaridade (PD) de 69\% entre as duas espécies, em relação às épocas de fundação de ninhos.

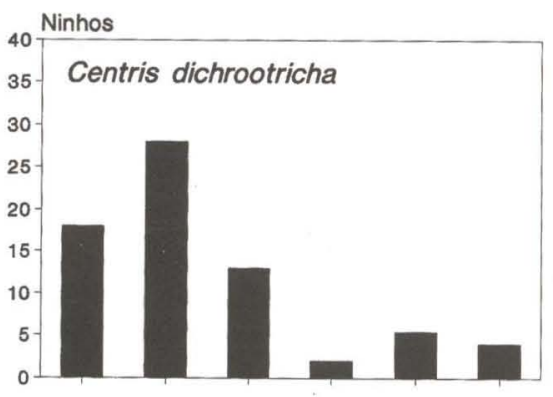

(A)

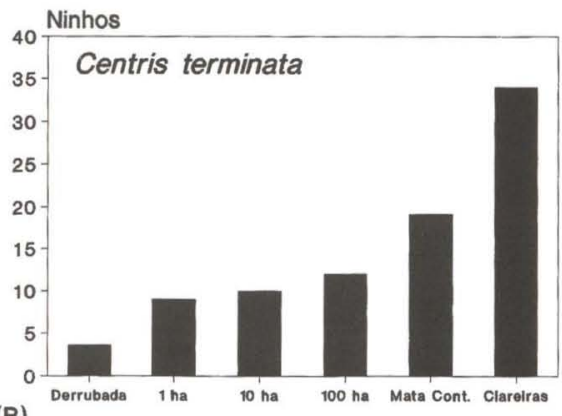

Fig. 2. (A) Precipitação $(\mathrm{mm})$ e temperatura $\left({ }^{\circ} \mathrm{C}\right)$ mensais para o período de junho de 1988 a junho de 1990 na região de estudo; (B) número total de ninhos fundados por espécies de Centris entre julho de 1988 a julho de 1989 (primeiro ano) e julho de 1989 a junho de 1990 (segundo ano); (C) número de ninhos fundados por $\mathrm{C}$. dichrootricha e $\mathrm{C}$. terminata entre julho de 1988 a junho de 1990.

\section{Arquitetura dos ninhos}

As fêmeas de $C$. terminata nidificaram em ninhos-armadilhas com $9,5 \mathrm{~mm}$ de diâmetro (71,3\% das fundações); $12,7 \mathrm{~mm}(20,2 \%)$ e 4,8 mm (8,5\%). Foram construídas de uma a seis células por ninho $(\mathrm{n}=94 ;$ moda $=4 ; 40,4 \%$ dos ninhos). Para a construção do ninho, as fêmeas usam fragmentos finos de madeira. As células têm formato oval, com largura variando entre 6,0 a 7,6 mm $(n=33)$ e comprimento variando entre 11,9 a $17,0 \mathrm{~mm}$; separadas por paredes com 0,6 a $1,4 \mathrm{~mm}$ de espessura. $\mathrm{O}$ comprimento médio foi maior nas células mais internas da cavidade, primeiras a serem construídas $(\mathrm{F}=12,132 ; \mathrm{p}<0,001)$ (Fig. 3). Em 35 ninhos 
examinados, oito possuíam além das células provisionadas, uma célula vestibular com comprimento variando entre 7,6 a 23,3 $\mathrm{mm}$. Com o mesmo material usado na construção das células, a fềmea faz uma parede na entrada do orifício para fechá-lo.

As fêmeas de $C$. dichrootricha nidificaram principalmente em ninhos-armadilhas com $12,7 \mathrm{~mm}$ de diâmetro ( $51,2 \%$ das fundações) e $9,5 \mathrm{~mm}$ (47,5\%). Apenas um ninho foi fundado em diâmetro de $4,8 \mathrm{~mm}$. Foram construídas de uma a sete células por ninho $(\mathrm{n}=58 ;$ moda $=5 ; 29,3 \%$ dos ninhos). As células são construídas com uma mistura de areia e óleo, possivelmente coletado nas flores. As células têm formato oval, com largura variando entre 6,9 a $8,4 \mathrm{~mm}(\mathrm{n}=33)$ e comprimento entre 9,0 a $14,8 \mathrm{~mm}$; são separadas por paredes com aproximadamente $1,5 \mathrm{~mm}$ de espessura. $\mathrm{O}$ comprimento médio foi maior nas células mais internas da cavidade $(\mathrm{F}=11,183 ; \mathrm{p}<0,001)$ (Fig. 3). Não foi constatada a presença de células vestibulares nos ninhos desta espécie. Ao finalizar a construção das células, a fêmea faz uma parede mais espessa servindo como tampa do ninho.

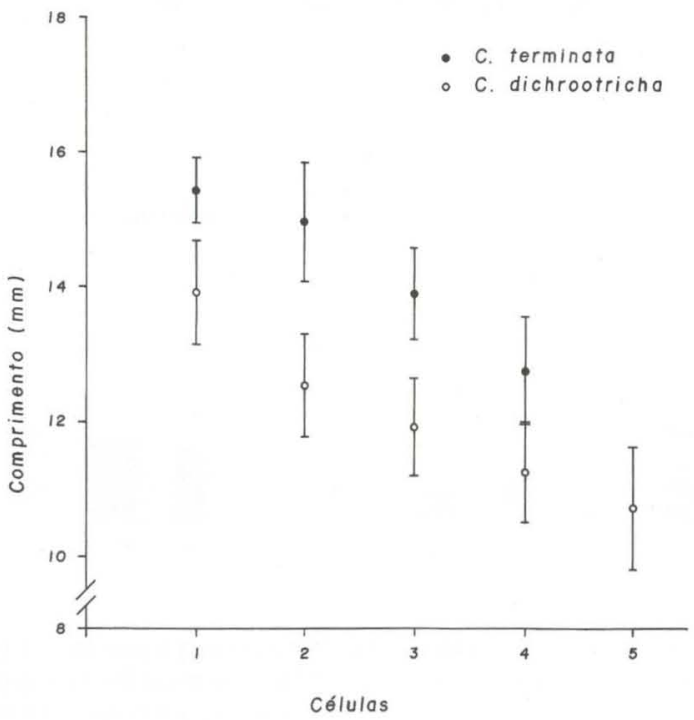

Fig. 3. Comprimento médio ( \pm intervalo de confiança a $95 \%$ ) das células, de acordo com sua posição, nos ninhos de $C$. terminata e $C$. dichrootricha. Células numeradas a partir da mais interna na cavidade (primeira a ser provisionada).

Foram fundados 16 ninhos de C. analis, sendo $12 \mathrm{em}$ cavidades com $4,8 \mathrm{~mm}$ de diâmetro e quatro em cavidades com $9,5 \mathrm{~mm}$, constituídos de duas a quatro células ( $n=13 ;$ moda $=4 ; 46 \%$ dos ninhos). As células, com comprimento variando entre 7 e $18 \mathrm{~mm}$, são construídas com fragmentos de madeira. O comprimento das células varia entre 7 e $18 \mathrm{~mm}$. Em dois ninhos foi constatada a presença de uma célula vestibular, uma com $3 \mathrm{~mm}$ e outra com $25 \mathrm{~mm}$ de comprimento.

Centris bicornuta fundou apenas três ninhos, todos em cavidades com 4,8 $\mathrm{mm}$ de diâmetro. As células são construídas com fragmentos de madeira e possuem 
comprimento variando entre 9 e $13 \mathrm{~mm}$. Em todos os ninhos havia cinco células provisionadas e em um deles foi constatada a presença de uma célula vestibular com $6 \mathrm{~mm}$ de comprimento.

\section{Emergência e razão sexual}

Emergiram 59 machos e 57 fêmeas de 42 ninhos de $C$. dichrootricha. De 56 ninhos de C. terminata emergiram 63 machos e 44 fêmeas. De 12 ninhos de C. analis emergiram 11 machos e 11 fềmeas e de dois ninhos de C. bicornuta emergiram um macho e duas fêmeas. Em nenhum desses casos a razão sexual foi diferente da proporção de 1:1 ( $p>0,05$; teste $\left.X^{2}\right)$. De 32 ninhos de $C$. dichrootricha, dos quais emergiram 2 ou mais adultos, $59 \%$ originaram adultos do mesmo sexo. Para $C$. terminata esse valor foi de apenas $25 \%$ (em 28 ninhos). Para cinco ninhos de $C$. analis, apenas 1 originou adultos do mesmo sexo.

\section{Parasitismo}

O número de células atacadas por insetos cleptoparasitas em ninhos de Centris spp. é mostrado na tabela II. Centris dichrootricha foi a espécie mais parasitada ( $30 \%$ dos ninhos e 32 células atacadas). Não foi constatado parasitismo nos ninhos de C. bicornuta. Tetraonyx Latreille, 1805 (Coleoptera, Meloidae) foi o cleptoparasita mais comum ( $70 \%$ das células atacadas). Em um ninho de $C$. terminata foi constatada a emergência de várias vespas parasitóides pertencentes à família Eurytomidae (Hymenoptera). Foi observada a emergência, em um mesmo ninho, de cleptoparasitas de diferentes espécies. Em um ninho de $C$. dichrootricha ocorreu a emergência de Coelioxys sp. e Tetraonyx sp. Em outro ninho, emergiram Tetraonyx sp. e Mesocheira bicolor (Fabricius, 1804). Em dois ninhos diferentes de C. terminata emergiram Tetraonyx sp. e Coelioxys sp. Em outro ninho, emergiram Tetraonyx sp. e uma espécie não identificada de Bombyliidae (Diptera).

Tabela II. Número de células parasitadas por insetos cleptoparasitas em ninhos provisionados por espécies de Centris spp. entre julho de 1988 e junho de 1990. (A) Tetraonyx sp. (Coleoptera, Meloidae), (B) Mesocheira bicolor (Hymenoptera, Anthophoridae), (C) Mesoplia sp. (Hymenoptera, Anthophoridae), (D) Coelioxys sp. 1 (Hymenoptera, Megachilidae), (E) Coelioxys sp. 2 (Hymenoptera, Megachilidae).

\begin{tabular}{lrrrrrrr}
\hline & \multicolumn{4}{c}{ Cleptoparasitas } & & $\begin{array}{c}\text { Porcentagem de } \\
\text { ninhos parasitados }\end{array}$ \\
\cline { 2 - 9 } \multicolumn{1}{c}{ Espécies } & A & B & C & D & E & Total \\
\hline Centris terminata & 15 & & 3 & 5 & 3 & 26 & 17 \\
Centris dichrootrich & 25 & 4 & & 3 & & 32 & 30 \\
Centris analis & 2 & & & & & 2 & 13 \\
\hline Total & 42 & 4 & 3 & 8 & 3 & 60 & \\
\hline
\end{tabular}

\section{DISCUSSÃO}

Os resultados mostraram que as espécies de Centris aqui estudadas mostraram diferentes preferências, em relação ao tipo de ambiente utilizado para nidificação. Para C. terminata o fato do número de fundações ter sido maior em clareiras criadas pela queda natural de árvores que em áreas de mata contínua mostra que 
essa espécie pode preferir esses ambientes mais iluminados e abertos que o sub-bosque da mata adjacente. Entretanto, o número de ninhos fundado em áreas desmatadas foi muito pequeno. Centris dichrootricha fundou mais ninhos em locais desmatados e pequenos fragmentos de mata. Isto sugere que $C$. terminata possa ser mais sensível à fragmentação da floresta do que $C$. dichrootricha. A fragmentação de florestas deve, portanto, exercer efeito negativo sobre suas populações. O oposto deve ocorrer para C. dichrootricha. A fragmentação de florestas deve, assim, afetar negativamente também espécies de plantas de polinização cruzada que dependam dessas abelhas para a produção de sementes.

Em um levantamento de Hymenoptera que nidificam em ninhos-armadilhas em um remanescente da Mata Atlântica (R. Pérez-Maluf \& L.A. de O. Campos, comunicação pessoal) constataram que Centris tarsata Smith, 1874 fundou mais ninhos em locais abertos ocupados por vegetação secundária.

$\mathrm{O}$ desmatamento e a fragmentação de florestas proporcionam o aparecimento de ambientes mais secos e com temperaturas mais elevadas (LOVEJOY et al. 1986).

FRANKIE et al. (1988) estudaram as populações de espécies de Centris que nidificam em cavidades preexistentes em uma floresta seca na Costa Rica. Constataram que, em relação à preferência por habitat, a maior parte dos ninhos foi fundada em ninhos-armadilhas colocados em locais sombreados. Nesses locais, em razão da queda de folhas das árvores, houve um aumento da temperatura onde os ninhos-armadilhas foram instalados, o que resultou em uma diminuição na taxa de fundação de ninhos.

É possível, portanto, que temperaturas altas possam ser limitantes à atividade de nidificação das fêmeas e ao desenvolvimento larval de espécies de Centris que nidificam em cavidades na madeira (FRANKIE et al. 1988).

De modo geral, as espécies de Centris fundaram mais ninhos a 8 e $15 \mathrm{~m}$ de altura ( $77 \%$ dos ninhos considerados). FRANKIE et al. (1988) instalaram ninhos-armadilhas a 0,5 e $2,5 \mathrm{~m}$ de altura em ambientes de mata e constataram que maior número de ninhos de Centris spp. foi fundado a $2,5 \mathrm{~m}$. É possível que em alturas próximas ao dossel, onde há mais disponibilidade de recursos florais, as condições microclimáticas sejam mais propícias à atividade de nidificação.

A maioria dos ninhos de Centris foram fundados entre agosto e janeiro, em ambos os anos de estudo. Padrão semelhante foi obtido para atividade de machos de Euglossini em iscas-odoríferas em florestas contínuas e fragmentos de mata nas áreas do PDBFF (BECKER et al. 1991). Não houve uma semelhança grande, em relação às épocas de maior atividade de fundação de ninhos entre $C$. dichrootricha e C. terminata. O padrão de distribuição de chuvas, ao longo dos meses, entre os dois anos foi diferente $\left(r_{s}=0,31 ;\right.$ G.L. $\left.=10 ; p>0,05\right)$. O padrão de atividade anual de fundação foi basicamente o mesmo para $C$. dichrootricha em ambos os anos. $\mathrm{O}$ mesmo não ocorreu com C. terminata. A atividade de nidificação de C. dichrootricha não apresentou correlação com a precipitação $\left(\mathrm{r}_{\mathrm{s}}=-0,38 ; \mathrm{G} . \mathrm{L} .=22 ; \mathrm{p}>0,05\right)$, o mesmo ocorrendo com C. terminata $\left(\mathrm{r}_{\mathrm{s}}=-0,05 ; \mathrm{G} . \mathrm{L} .=22 ; \mathrm{p}>0,05\right)$.

$\mathrm{O}$ conhecimento sobre a arquitetura dos ninhos de espécies de Centris em cavidades preexistentes é escasso. FRANKIE et al. (1988) relataram algumas características dos ninhos de seis espécies de Centris, entre elas C. analis e C. bicornuta. 
O material usado na construção das células por estas duas espécies é semelhante ao por nós observado. As demais espécies usaram areia na construção das células, tal como C. dichrootricha.

C. dichrootricha foi a espécie que sofreu maior parasitismo e Tetraonyx sp. o parasita mais freqüente. Não existe nenhum registro deste coleóptero atacando ninhos de espécies de Centris. Espécies de Coelioxys Latreille, 1809, Mesoplia Lepeletier, 1841 e Mesocheira Lepeletier \& Serville, 1825 também parasitaram os ninhos. Embora Coelioxys tenha sido relatada parasitando ninhos, principalmente, de espécies de Megachile Latreille, 1802, MitCHELL (1973) e BATRA \& SCHUSTER (1977) também relataram uma espécie não identificada de Coelioxys parasitando ninhos de espécies de Centris que nidificam no chão. Espécies de Mesoplia têm sido relatadas parasitando ninhos de espécies de Centris que nidificam no chão (COVILLE et al. 1983; VINSON et al. 1987). VINSON \& FRANKIE (1991) observaram fêmeas de Mesoplia sobrevoando próximas de ninhos terrestres de C. aethyctera Snelling, 1974 na Costa Rica. Contudo, não observaram emergência de adultos de Mesoplia nos ninhos.

AGRADECIMENTOS. Ao PDBFF pelo apoio logístico e financeiro. Esta é a publicação n 263 do PDBFF. Ao Dr. Pe. Jesus Santiago Moure pela identificação das espécies de abelhas.

\section{REFERÊNCIAS BIBLIOGRÁFICAS}

Alcock, J.; C.E. JoneS \& S.L. BuchmanN. 1976. The nesting behavior of three species of Centris bees (Hymenoptera: Anthophoridae). Jour. Kans. Entomol. Soc. 49 (4): 469-474.

BAtra, S.W. \& J.C. Shuster. 1977. Nests of Centris, Melissodes and Colletes in Guatemala (Hymenoptera: Apoidea). Biotropica 9 (2): 135-138.

BeCKer, P.; J.S. Moure \& F.J.A. PERALTA. 1991. More about euglossine bees in amazonian forest fragments. Biotropica 23 (4b): 586-591.

BENETT, F.D. 1964. Notes on the nesting site of Centris derasa (Hymenoptera: Apoidea). Pan-Pac. Entomol. 40 (2): 125-128.

BIERREGAARD JR., R.D. \& T. LOVEJOY. 1988. Birds in Amazonian forest fragments: effects of insularization, p.1564-1579. In: H. QUELlet (Ed.). Acta XIX Cong. Int. Ornith. Ottawa, Univ. of Ottawa Press, 1409p.

CHANDleR, L.; J.A.F. BARRIGossi \& E.B.S. Diaz. 1985. The first definitive host record for Leucospis cayennensis Westwood (Hymenoptera: Leucospidae). Rev. Ceres 32 (180): 170-174.

Coville, R.E.; G.W. FrankiE \& S.B. Vinson. 1983. Nests of Centris segregata (Hymenoptera: Anthophoridae) with a review of the nesting habits of the genus. Jour. Kans. Entomol. Soc. 56 (2): 109-122.

Coville, R.E.; G.W. FrankiE; S.L. BuchmanN; S.B. Vinson \& H.J. Williams. 1986. Nesting and male behavior of Centris heithausi (Hymenoptera: Anthophoridae) in Costa Rica with chemical analysis of the hindleg glands of males. Jour. Kans. Entomol. Soc. 59 (2): 325-336.

FranKIE, G.W.; S.B. Vinson; L.E. NEWSTROM \& J.F. BARTHELl. 1988. Nest site and habitat preferences of Centris bees in Costa Rican dry forest. Biotropica 20 (4): 301-310. 
Frankie, G.W.; L. NewSTROM; S.B. VINSON \& J.F. BARTHELL. 1993. Nesting-habitat preferences of selected Centris bee species in Costa Rica Dry Forest. Biotropica 25 (3): 322-333.

GotTSBERGER, G.; J.M.F. DE CAMARGO \& I. SILBERBAUER-GotTSBERGER. 1988. A bee-pollinated tropical community: the beach dune vegetation of Ilha de São Luís. Maranhão, Brazil. Bot. Jahrb. Syst. 109 (4): 469-500.

LOVEJOY, T.E.; J.M. RANKIN; R.O. BIERREGAARD JR.; A.B. RYLANDS; J.R. MALCON; C.E. Quintela; L. HarPer; K.S. Brown JR.; A.H. POWELl; G.V.N. POWELL; H.O.R. SCHUBART \& M.B. HAYs. 1986. Edge and other effects on isolation on Amazon forest fragments, p.257-285. In: M.E. Soule (Ed.). Conservation biology: the science of scarcity and diversity. Sunderland, Massachusetts, Sinauer, 584p.

LUDWIG, J.A. \& J.F. REYNOLDS. 1988. Statistical Ecology: a primer on methods and computing. New York, John Wiley, 337p.

MiCHENER, C.D. \& R.B. LANGE. 1958. Observation on the ethology of neotropical anthophoridae bees (Hymenoptera: Apoidea). Univ. Kansas Sci. Bull. 39: 69-96.

MitCHELl, T.B. 1973. A sugeneric revision of the bees of the genus Coelioxys of the Western hemisphere (Hymenoptera: Megachilidae). Raleigh, North Carolina, North Carolina State University, 129p.

Pickel, B. 1928. Contribuição para a biologia de Centris sponsa e Acanthopus excellens (Hymen.). Bol. Biol. São Paulo 14: 135-143.

PIRES, J.M. \& G.T. PRANCE. 1985. The vegetation types of the brazilian amazon, p.109-145. In: G.T. PRANCE \& T.E. LOVEJOY (Ed.) Amazonia (Keyenviroments). Oxford, England, Pergamon Press, 442p.

RAU, P. 1943. Notes on the nesting habits of certain social and solitary bees of Mexico. Ann. Entomol. Soc. Amer. 36: 641-646.

RAW, A. 1984. The nesting biology of nine species of Jamaican bees (Hymenoptera). Revta bras. Ent. 28 (4): 497-506.

RIBEIRO, M. DE N.G. \& J. ADIS. 1984. Local rainfall variability -a potential bias for bioecological studies in the Central Amazon. Acta Amazonica 14: 159-174.

VeZeY-FitZGERALD, D. 1939. Observations on bees (Hym.: Apoidea) in Trinidad. Proc. Royal Ent. Soc. London (A) 14: 107-110.

VINSON, S.B. \& G.W. FRANKIE. 1977. Nests of Centris aethyctera (Hymenoptera: Apoidea: Anthophoridae) in dry forest of Costa Rica. Jour. Kans. Entomol. Soc. 50 (2): 301-311.

1988. A comparative study of the ground nests of Centris flavifrons and Centris aethiocesta (Hymenoptera: Anthophoridae). Entomol. Exp. Appl. 49: 181-187.

1991. Nest variability in Centris aethyctera (Hymenoptera: Anthophoridae) in response to nesting site conditions. Jour. Kans. Entomol. Soc. 64 (2): 156-162.

VInSON, S.B.; G.W. FrankIE \& R.E. CoviLle. 1987. Nesting habits of Centris flavofasciata Friese (Hymenoptera: Apoidea: Anthophoridae) in Costa Rica. Jour. Kans. Entomol. Soc. 60 (2): 249-263.

Recebido em 28.V.1998; aceito em 29.X.1999. 